\title{
E-commerce de moda: uma reflexão sobre os cenários atuais e futuros
}

Fashion e-tail: a reflection on current and future scenarios

BOZZI, Carolina; M. Sc.; PUC-Rio

carolbozzi@gmail.com

MONT'ALVÃO, Claudia; Titulação; PUC-Rio

cmontalvao@puc-rio.br

\section{Resumo}

Este artigo propõe discutir o estado-da-arte, os modos de interação, as interfaces e as técnicas de pesquisa do comércio eletrônico. Verifica-se que com a introdução de novas tecnologias surgem usuários/consumidores com mais acesso à informação e mais exigentes. A "hiperconectividade" possibilita a existência de novas formas de interagir com o e-commerce. O processo de compra do usuário não acontece on-line ou off-line, as barreiras entre os mundos real e digital estão cada vez mais entrelaçadas, dando espaço às estratégias varejistas omnichannel, oferecendo uma experiência do usuário mais completa. Este artigo considera que os modos on-line e off-line sejam complementares Estas são as premissas pelas quais serão discutidas as tendências atuais e as possibilidades futuras para e-commerce.

Palavras Chave: e-commerce: UX; usabilidade.

\section{Abstract}

This article proposes a discussion about the e-commerce state-of-the-art, its interaction modes, interfaces and research techniques. It has been verified that with the introduction of new technologies and the emergence of users / consumers with more access to information who are increasingly more demanding. "Hyperconnectivity" enables the existence of new ways of interacting with e-commerce. The user decision-making process does not happen online or offline, the barriers between the real and digital worlds are blurring, giving room to the omnichannel retail strategies that offer a more complete UX. The online and offline are actually foundo to be complementary. With this scenario setting the current trends and future possibilities for $e$ commerce will be discussed.

Keywords: ecommerce; UX; usability. 


\section{Introdução}

Desde a introdução do comércio eletrônico, o e-commerce, nos anos dos 1990, o varejo tem sido alvo de constantes mudanças em consequência das novas dinâmicas de mercado impostas por novas tecnologias e demandas do consumidor que está mais bem informado e exigente. Ele busca não somente adquirir produtos, mas ter uma experiência de compra.

O comércio eletrônico de vestuário ocupa os primeiros lugares das categorias de produtos mais vendidos mundialmente. Segundo um relatório da The Nielsen Company (2017), 58\% dos usuários globais declaram já terem adquirido algum produto desta categoria em sítios de ecommerce, seguido de $55 \%$ de produtos relacionados a viagens e $43 \%$ de produtos de $\mathrm{TI}$ e mobile. A despeito dos excelentes índices de performance, o comércio eletrônico de roupas propõe diversos desafios para quem vende e para quem compra, existe uma dificuldade em comunicar por meio de uma tela alguns atributos dessa categoria de produtos como a textura e o caimento do tecido. $\mathrm{O}$ objetivo deste artigo é discutir as estratégias atuais abordadas pelos varejistas com o intuito de dar suporte ao processo decisório de compra usuário/consumidor para facilitar e atender novas demandas.

A primeira parte do artigo exporá o panorama atual do e-commerce. Serão apresentados os principais números mundiais e nacionais e uma visão geral do cenário do segmento de moda e acessórios. Em seguida, examinar-se-ão alguns dos desafios, enfrentados pelos consumidores de moda ao comprar produtos on-line e as soluções que estão sendo propostas para melhorar a experiência do usuário, a UX. Por fim, com base no cenário atual, serão propostos possíveis caminhos e tendências para o segmento do comércio eletrônico de vestuário.

Para fins de esclarecimento, o usuário é a pessoa que interage com um produto/sistema (ABNT, 2002). Este termo será utilizado em referência a um indivíduo que não esteja inserido em um âmbito de compras. Consumidor é uma pessoa que tem uma necessidade ou um desejo, faz uma compra e depois descarta o produto durante as três fases do processo de compra (SOLOMON, 2011). Este termo será adotado, quando for feito uma referência ao sujeito em um âmbito de consumo.

\section{Cenário atual do e-commerce}

Em 2016 o número total de usuários da internet em nível mundial chegou a 3,5 bilhões, (THE NIELSEN COMPANY, 2017), destes quase 1,5 bilhão compram em sítios de comércio eletrônico, denominados de e-shoppers (ECOMMERCE FOUNDATION, 2016). No Brasil, 81,3 milhões dos usuários acessam a internet por meio de um desktop ou laptop. 60,4 milhões usam smartphone e 11,8 milhões são usuários de tablet para acessar a web (COMSCORE, 2016). Segundo a 37ạ edição do Webshoppers, 55,5 milhões de brasileiros fizeram pelo menos uma compra virtual em 2017, um aumento de $15,3 \%$ em relação à $2016,49,4 \%$ são homens e $50,6 \%$ mulheres (E-BIT, 2018). Esta distribuição é bastante diferente, quando se considera o segmento de moda e acessórios isoladamente, no qual as mulheres são a maioria, representam $66,7 \%$ dos consumidores (ATLAS, 2017) (Figura 1). A idade média dos usuários de internet é de 42.2 anos, com uma concentração maior (38\%) na faixa dos 35 a 49 anos (E-BIT, 2018). O faturamento do ecommerce, em 2017, no Brasil, foi de $\mathrm{R} \$ 47,7$ bilhões, oriundos de 111,2 milhões de pedidos. 27, $3 \%$ destes pedidos foram feitos em dispositivos móveis e o Sudeste é o maior mercado no País (EBIT, 2018). 


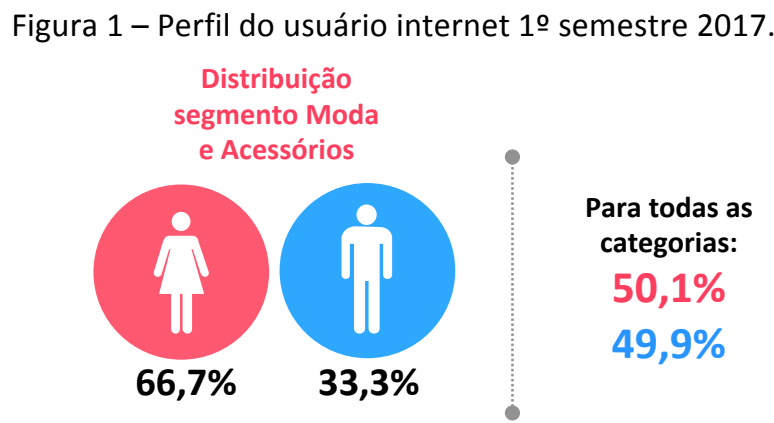

Fonte: (ATLAS, 2017; E-BIT, 2017).

O Webshoppers (E-BIT, 2018) mostra que as categorias mais populares em volume de pedidos são: 1으 moda e acessórios (14,2\%), 2o saúde cosméticos e perfumaria (12,0\%) 3은 eletrodomésticos $(10,8 \%)$. Porém, quando se considera o volume de faturamento, o segmento de moda e acessórios não aparece nos três primeiros lugares, dando espaço para: 10 telefonia/celulares (21,2\%) 2 e eletrodomésticos (19,3\%) e 30 eletrônicos $(10,0 \%)$. Estes números podem indicar que os consumidores investem menos dinheiro nestes produtos, sugerindo a existência de um risco percebido maior ao comprar roupas on-line. As categorias mais populares, em volume de pedidos no $m$-commerce, são bem similares ao número geral do e-commerce: 1 은 moda e acessórios (15\%), 2o casa e decoração (14\%) e 3o saúde cosméticos e perfumaria $(10,6 \%)$ (E-BIT, 2017).

\section{0 processo decisório do consumidor e os modos de interação do e-commerce}

O entendimento do processo de tomada de decisão do consumidor ajuda na compreensão dos modos de interação presentes no e-commerce. 0 modelo tradicional normalmente começa com o reconhecimento de uma necessidade, seguido da busca de informações acerca de um produto ou serviço, avaliação de alternativas, a decisão de compra (ou não) de um determinado produto ou serviço e por fim, o comportamento pós-compra (KATAWETAWARAKS; WANG, 2011; KOTLER, 2000; SOLOMON, 2011). Kotler, Keller (2016) e Solomon (2011) explicam as diferentes etapas do processo decisório de compra: O processo de compra começa quando um consumidor reconhece um problema ou necessidade desencadeados por estímulos internos ou externos.

A busca por informações é a segunda etapa do processo quando os consumidores procuram por dados apropriados acerca do produto ou serviço desejado. Classificam-se dois níveis de engajamento na busca; o primeiro é o chamado de atenção aumentada. Neste nível, o consumidor torna-se mais receptivo às informações. No segundo nível, o consumidor passa a realizar uma pesquisa de informações ativa: procura materiais escritos, como artigos e manuais, pede informações para amigos, acessa sítios na web e visita lojas físicas para saber mais. As principais fontes de informação procuradas por consumidores são classificadas em quatro grupos:

Pessoal: família, amigos, vizinhos, conhecidos;

Comercial: publicidade, sites, e-mails, vendedores, revendedores, embalagens, displays;

Público: meios de comunicação social, redes sociais; e

Experiencial: manipulação e uso do produto.

A quantidade de informação e influência destas fontes varia de acordo com a categoria do 
produto e as características do comprador. Mesmo no ambiente on-line, em que não existe contato tátil com o produto antes da compra, o consumidor pode dirigir-se à loja física antes de realizar uma compra digitalmente e isto ser um fator determinante no seu processo decisório.

Avaliação das alternativas - uma vez reunidas e avaliadas as opções de um produto ou serviço, eventualmente uma delas tem de ser a escolhida. As regras de decisão que orientam as escolhas variam de estratégias muito simples e rápidas a processos complicados, exigentes de muita atenção e processamento cognitivo. Não existe um processo único usado por todos os consumidores, há vários. Primeiro, o consumidor está tentando satisfazer uma necessidade. Em seguida, está procurando benefícios específicos de um produto ou serviço. E por último, o consumidor vê cada produto como um conjunto de atributos com diferentes habilidades para oferecer estes benefícios e satisfazer sua necessidade. Os atributos de interesse para os compradores variam de acordo com o produto, a título de exemplo:

1. Hotéis - localização, limpeza, atmosfera, preço;

2. Lavagem bucal - cor, eficácia, capacidade de matar os germes, sabor, preço;

3. Pneus - segurança, vida do piso, qualidade do passeio, preço.

Os consumidores prestarão mais atenção aos atributos que oferecem os benefícios procurados.

Decisão de compra - Na fase de avaliação, o consumidor escolhe entre diversas marcas de sua preferência e pode formar a intenção de comprar sua marca preferida. Ao executar uma intenção de compra, o consumidor pode fazer até cinco decisões complementares: marca (marca A ou B), revendedor (revendedor 1 ou 2), quantidade (um, dois ou três), ocasião (fim de semana) e método de pagamento (cartão de crédito).

Comportamento pós-compra - Após a compra o consumidor pode sentir-se satisfeito ou insatisfeito com a sua aquisição. No ambiente on-line ele só terá contato com o produto quando este for-lhe entregue. A satisfação é uma função da proximidade entre as expectativas e o desempenho percebido pelo produto. Se o desempenho não atende às expectativas, o consumidor está insatisfeito; se atende às expectativas, o consumidor está satisfeito; se exceder as expectativas, o consumidor está encantado. Esses sentimentos fazem a diferença na experiência total do usuário. Quanto maior a lacuna entre as expectativas e o desempenho, maior a insatisfação.

O comportamento do consumidor é influenciado por três fatores: culturais (cultura, subcultura e classe social), sociais (grupos de referência, família e papéis sociais e status) e pessoais (idade, estágio no ciclo de vida, ocupação, circunstâncias econômicas, estilo de vida, personalidade e auto-concepção). A pesquisa sobre aqueles fatores pode fornecer pistas para alcançar e atender os consumidores de forma mais eficaz. É essencial conhecê-los bem.

O processo de compra não é necessariamente seguido na ordem apresentada acima; é possível que etapas sejam puladas, repetidas, e que os canais utilizados sejam tanto on-line quanto off-line. Entre os canais on-line estão sites, e-mails recebidos, mídias sociais, avaliações online de outros consumidores, entre outros (Figura 2). Os canais off-line incluem a loja física, revistas e jornais, recomendações de vendedores, boca-a-boca de amigos e conhecidos entre outros (THE NIELSEN COMPANY, 2017).

A oferta de canais on-line e off-line está mudando a dinâmica do processo decisório, principalmente, a forma como se buscam informações sobre produtos e serviços. Com a crescente 
popularidade do uso de smartphones e, consequentemente, o tempo em que se está conectado à internet molda os novos hábitos de compra. O resultado desta "hiperconectividade" são consumidores muito bem informados e exigentes que utilizam diferentes canais para tomar uma decisão de compra.

Fig. 2 - Interações do e-commerce.

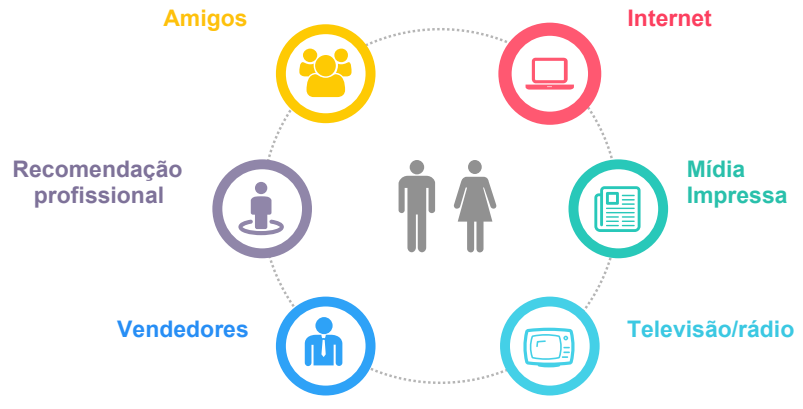

Fonte: elaborado pela autora.

\section{Omnichannel: estratégia contra a incerteza do consumidor ao comprar on-line}

Dependendo do canal utilizado, os consumidores podem não ter informações completas antes de fazer uma compra. Por exemplo: percebem que o ajuste de um produto de vestuário não é totalmente observado antes da compra quando se utilizam do canal on-line, uma vez que não é possível fazer uma inspeção completa do produto. (ANDERSON; HANSEN; SIMESTER, 2009). Uma análise prévia torna-se especialmente importante para os consumidores, quando o produto possui atributos não-digitais. Segundo Lal e Sarvary (1999), os atributos não-digitais são aqueles que são difíceis de serem comunicados por canais on-line e podem representar incertezas significativas para alguns consumidores, como por exemplo a textura do tecido de uma camisa. Os atributos digitais dos produtos são aqueles facilmente comunicados on-line sem perda de informação como o preço ou o tamanho de um livro. Portanto, a incapacidade dos consumidores de tocar e sentir produtos com atributos não digitais antes de comprar pode: atuar como dissuasor para a compra; e aumentar os custos operacionais se retornarem os produtos após experimentarem; uma discrepância entre o esperado e produto entregue. (LAL; SARVARY, 1999)

Uma vez que os mundos real e virtual concorrem entre si; mas, ao mesmo tempo, se complementam, é natural se entrelaçarem, onde um deles for mais fraco, o outro será mais forte (BELL, DAVID, 2016). Impulsionados pelo uso de novas tecnologias no varejo, os hábitos e expectativas de compra também estão mudando. A demanda por experiências que unam o mundo real com o mundo digital é cada vez maior (BELL, 2016, apud Kim Snow ${ }^{1}$, 2013). Um novo consumidor, usuário de múltiplos dispositivos e múltiplas telas, emergiu: ele tem acesso fácil à informação e exige marcas que ofereçam uma estratégia omnichannel possibilitando a união do on-line com o off-line proporcionando uma UX integrada (JUANEDA-AYENSA; MOSQUERA; MURILLO, 2016).

\footnotetext{
${ }^{1}$ Diretora de criação do Google em Nova York) - Seminar on possibilities, Google Brand Health Summit 2013.
} 
O Norman e o Nielsen [s.d.]) definem que UX abrange todos os aspectos da interação do usuário final com a empresa, seus serviços e produtos. O Kuniavsky (2010) define a UX como o conjunto de percepções do usuário final ao interagir com um produto ou serviço. Essas percepções incluem a eficácia, a eficiência e a satisfação, além da qualidade do relacionamento com a entidade responsável pelo produto ou serviço.

A UX omnichannel aborda não somente a interação do usuário com o produto e a marca como também sua transição entre os diversos canais oferecidos e sua habilidade de proceder suavemente entre as diversas etapas de interação (FLAHERTY, 2016). Os consumidores esperam que um serviço ou experiência seja consistente, uniforme e integrado, independente do utilizado; estão dispostos a alternar entre os diferentes canais oferecidos (loja tradicional, on-line e móvel) para efetuar uma compra dependendo de suas preferências, de sua situação atual, da hora do dia ou da categoria do produto (JUANEDA-AYENSA; MOSQUERA; MURILLO, 2016).

A estratégia omnichannel é uma evolução do crosschannel e do multichannel. O multichannel é a oferta de mais de um canal de contato com o consumidor, sem haver uma preocupação de integração entre os canais, são independentes e não existe a possibilidade do consumidor utilizá-los de forma integrada. Por exemplo: uma pessoa pode adquirir um produto em lojas físicas, sítios de e-commerce, e-mail ou aplicativos móveis (BECK; RYGL, 2015).

O crosschannel é o conjunto de atividades envolvidas na comercialização de produtos ou serviços por mais de um canal (loja física, catálogo, telefone, loja online e loja móvel). Ele propõe uma forma de interação entre canais, que ajude a suavizar a experiência dos usuários, mais de um canal pode ser usado para a mesma transação. Eles deixam de ser concorrentes e tornam-se complementares. O consumidor pode interagir parcialmente com os canais e/ou o varejista controla esta integração (BECK; RYGL, 2015).

Entende-se que a estratégia omnichannel ofereça uma interação e uma transição entre canais sem causar desconforto para o usuário; ela tem de acontecer de forma integrada. Existe uma sinergia no gerenciamento dos inúmeros canais disponíveis e dos pontos de atendimento do consumidor de forma que sua experiência e desempenho seja otimizada. Os canais interagem uns com os outros e são usados simultaneamente (VERHOEF; KANNAN; INMAN, 2015).

Frequentemente, os usuários não completam uma atividade em uma única sessão ou em um único canal. Flaherty (2017) aponta algumas das razões mais comuns pelas quais os usuários se alternam entre os canais: 1) quando existe uma interrupção ou mudança externa no contexto, em algumas situações, os usuários não conseguem completar uma atividade de uma só vez porque são interrompidos. 2) A tarefa é mais adequada para outro canal. Os usuários geralmente mudarão de canal porque sua atividade tornou-se muito trabalhosa para o canal atual e mudar parece mais eficaz ou eficiente. 3) A natureza da atividade exige isso. Algumas atividades exigem que os usuários utilizem diversos canais para completá-las.

A autora supracitada, lista 5 componentes da UX omnichannel que juntos criam uma estratégia de bem-sucedida, que deve ser:

Consistente: proporcionar uma experiência consistente, coesa e familiar em todos os canais Otimizada: criar experiências de canais individuais mais adequadas para determinado dispositivo, levando em conta as restrições do canal e o contexto de uso;

Seamless (sem emendas, integrada): tornar as transições entre os canais fácil e auxiliar os usuários a retomar uma tarefa do mesmo ponto em que pararam ao mudarem de um canal para outro; 
Orquestrada: guiar os clientes de maneira proativa por suas jornadas individuais com interações e mensagens personalizadas no momento certo;

Colaborativa: enriquecer a jornada do usuário, permitindo os aproveitar múltiplos canais simultaneamente para melhorar sua experiência geral.

Os usuários conectados utilizam múltiplas telas e canais, o dispositivo utilizado geralmente é definido por onde estamos, o que quer-realizar-se e o tempo necessário (GOOGLE, 2012) ou disponível. A usabilidade está no centro desta equação de múltiplas telas, sua essência está no acordo entre interface, usuário, tarefa e ambiente (CYBIS; HOLTZ; FAUST, 2010). A usabilidade é definida pela A ISO 9241-11 como: a "medida na qual um produto pode ser usado por usuários específicos com eficácia, eficiência e satisfação em um contexto específico de uso" (ABNT, 2002). Portanto, fazer uma ligação entre UX e usabilidade deveria permitir aos designers aplicarem os princípios de usabilidade na busca de uma UX positiva (MOCZARNY; DE VILLERS; VAN BILON, 2012). É importante ressaltar que a UX não é igual à usabilidade; a usabilidade percebida pelo usuário normalmente é um aspecto que contribui com a UX como um todo (ROTO et al., 2010).

A possibilidade de comprar em um contexto omnichannel apresenta inúmeras vantagens: ela afeta a influência que a necessidade de segurança exerce, um fator importante principalmente no ambiente digital, ao permitir a opção de pagamento tradicional. Esta possibilidade diminui o efeito do risco percebido no comércio eletrônico. Desta forma a adoção de uma estratégia omnichannel oferece a oportunidade de atrair consumidores mais conservadores que têm uma percepção de risco mais acentuada (JUANEDA-AYENSA; MOSQUERA; MURILLO, 2016). Os principais motivos pelos quais alguns consumidores ainda preferem comprar em lojas físicas expressa-se pela vontade de querer ter um contato direto com o produto, como indica um relatório sobre o varejo da KPMG (2017) (Figura 3).

Existe claramente um problema na comunicação dos atributos não digitais das peças de vestuário. Somada à dificuldade de transmitir-se determinadas características de uma roupa, como a textura do tecido, está a falta de padronização das medidas. O resultado são consumidores insatisfeitos e uma UX negativa. Os varejos de moda estão recorrendo a diversos recursos, como as estratégias omnichannel e ferramentas tecnológicas para tentar resolver de forma agressiva um problema que além de prejudicial à indústria também o é para a UX; a devolução. A incapacidade de interagir fisicamente com o produto resultou em taxas de devolução que em alguns casos chegaram a 50\% em 2015 (SHOPIFY, 2016). Ao oferecer uma ampla variedade de opções de canais, o revendedor pode compensar os pontos fracos inerentes a cada alternativa (BELL, DAVID R.; GALLINO; MORENO, 2016). Atualmente existem inúmeras soluções que os varejistas estão adotando para oferecer opções de canais para dar suporte ao processo decisório de compra de roupas do consumidor on-line, algumas, mais populares estão listadas abaixo:

Popup stores e Guide shops - Uma pop up store é uma loja temporária, uma alternativa para varejistas on-line atraírem o público-alvo para o meio físico. Uma guide shop é uma loja física onde os varejistas on-line expõem o produto, mas a compra é digital. Estes dois formatos de loja permitem ao usuário ter um contato físico com os produtos oferecidos digitalmente.

Try-before-you-buy (Experimentar antes de comprar) - Algumas empresas, como a Amazon.com, permitem que o usuário faça o pedido, receba o produto em casa e o experimente produto antes de efetuar o pagamento.

See-now, Buy-now (Viu, comprou) - os consumidores podem comprar as peças que são desfiladas nas passarelas nas semanas de moda imediatamente após os desfiles. 
Fig. 3 - Razões por que um usuários compram em lojas físicas

Eu quero ver/tocar o produto primeiro

Eu quero experimentar o produto Receio do produto parecer diferente

Demora na entrega Frete muito caro

Produto é muito valioso para comprar on-line

Gosta da experiência de ir à loja Tem de ir à loja de qualquer maneira

Quer verificar a autenticidade

Processo de devolução é muito complicado

Não confiam na segurança on-line

Querem falar com um vendedor

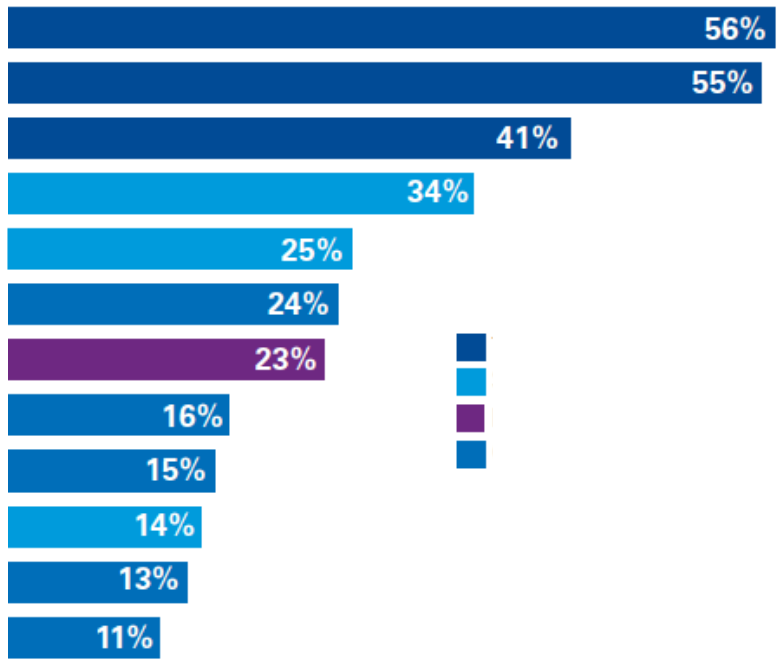

Fonte: (KPMG, 2017).

\section{Interfaces digitais e não digitais do e-commerce}

As interfaces digitais são um meio de comunicação entre um usuário e um sistema; atualmente, usuários passam $90 \%$ do tempo total dedicado às mídias de comunicação $(4,4$ horas/dia em média) diante de alguma tela, smartphones, desktops/laptops, tablets ou/e televisores (GOOGLE, 2012) executando diversas tarefas. Para efetuar compras on-line os desktops e laptops ainda são as interfaces mais utilizadas (Figura 4). O uso dos smartphones para efetuar compras on-line está crescendo. Na ultima Black Friday, representou 26,5\% em volume financeiro das compras realizadas, uma alta de $41,5 \%$ ante 2016, no Brasil (dado divulgado pela E-bit) (ECOMMERCE NEWS, 2017). Também são os principais dispositivos para iniciar o processo decisório de compra; são amplamente utilizados para pesquisar preços e buscar informações sobre produtos (GOOGLE, 2012).

Além das interfaces digitais, frequentemente em forma de telas, existem aquelas que não o são, o usuário/consumidor ainda pode ter como interface os vendedores nas visitas às lojas físicas, revistas e jornais durante o processo decisório de compra, como mencionado anteriormente. Entretanto, o surgimento e desenvolvimento de alguns tipos de tecnologia podem introduzir novas interfaces no e-commerce, apresentadas no próximo item.

Fig. 4 - Dispositivo preferido para comprar on-line. 


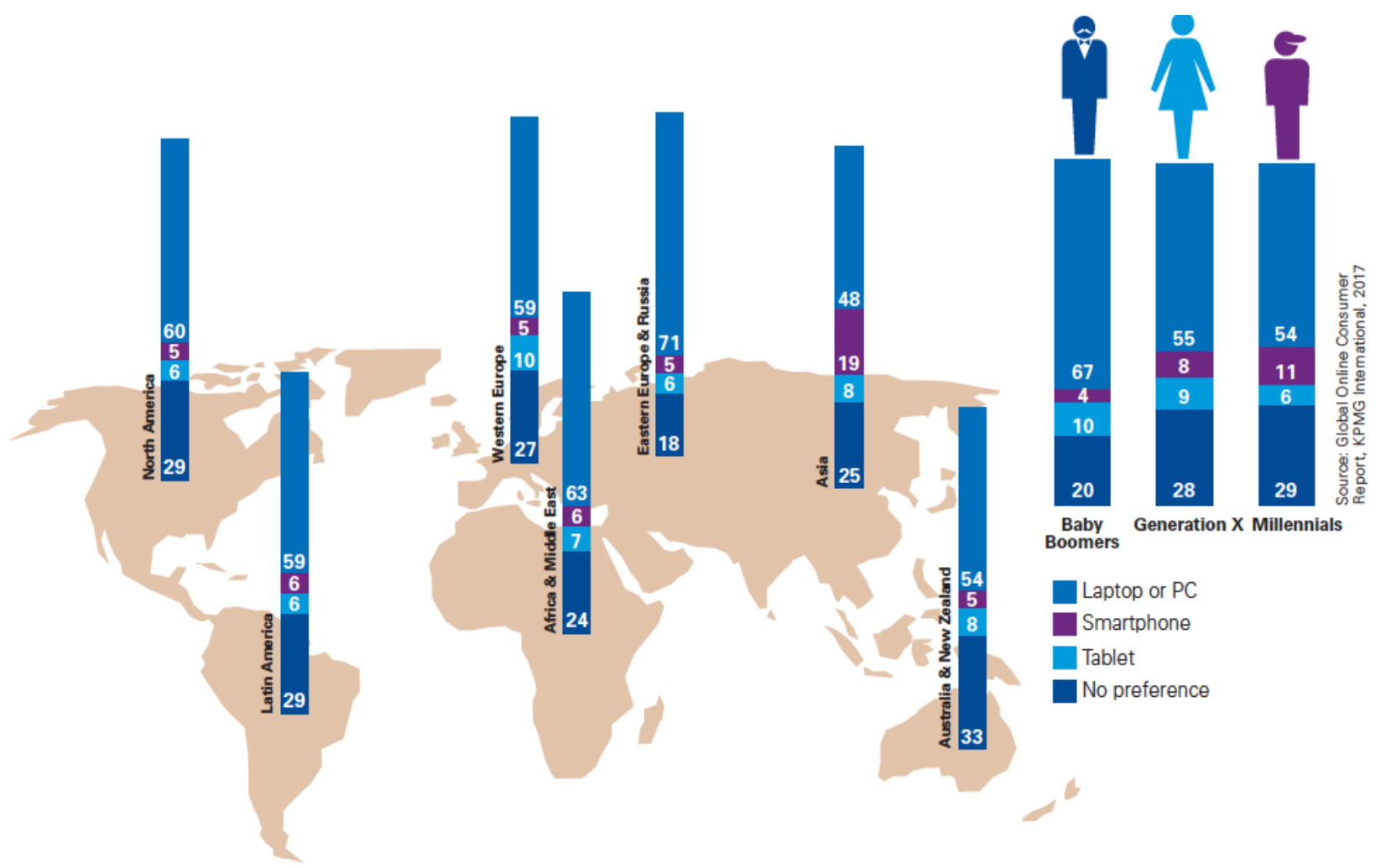

Fonte: (KPMG, 2017).

\section{Interfaces futuras do e-commerce de vestuário}

O ambiente digital e algumas tecnologias emergentes são as ferramentas para um futuro brilhante para o dimensionamento de roupas personalizadas em massa. $O$ escaneamento corporal pode ser facilmente acessível em cartões inteligentes que os consumidores carregam, usam online e compartilham com fabricantes de roupas, ou em uma localização central on-line acessível por meio de senhas (LOKER, 2007).

As compras on-line representam uma crescente parcela no mercado do vestuário e os bodyscanners 3D estão substituindo o processo manual de tirar medidas usando uma fita métrica. Atualmente já é possível fazer uma simulação de como uma peça de roupa, que se vai vestir, utilizando-se modelos digitais. Pode determinar-se o tipo de corpo, estilo de cabelo e forma facial. Os configuradores de produtos on-line oferecem múltiplas opções de estilo para experimentação e exploração de design personalizado. No futuro, os avanços proporcionados pelas possibilidades dos bodyscanners aliados à realidade aumentada e a internet das coisas, é possível que as simulações de ajuste de peças ao corpo sejam feitas utilizando-se informações individuais e possam ser feitas utilizando-se a câmera de um desktop/laptop ou de um smartphone. Uma vez que as ferramentas de visualização sejam aperfeiçoadas para que se possa usar o escaneamento corporal, será possível avaliar o tamanho e caimento on-line (LOKER, 2007).

Loker (2007) especula que os consumidores poderão desenhar roupas para eventos específicos e ajustá-las de acordo com preferências pessoais ou funcionais. Os configuradores de produtos poderão oferecer duas, três ou mais preferências de ajuste, tais como: justo, regular e 
solto que atualmente são oferecidos por alguns revendedores de roupas customizadas on-line (por exemplo, Lands' End). Um benefício adicional seria experimentar a roupa virtualmente em seu corpo escaneado. $\mathrm{O}$ ajuste individual poderia ser visto on-line e o consumidor poderia selecionar o ajuste mais adequado a seu gosto pessoal.

A Figura 4 ilustra alguns destes recursos que utilizam a realidade aumentada já existentes em lojas de vestuário que simulam como a roupa vai ficar no corpo. Estes mesmos simuladores, com o avanço da tecnologia podem ser trazidos para uma esfera mais pessoal; a tela do smartphone ou desktop/laptop. No entanto, embora esta imagem simulada possa fornecer ao cliente uma ideia mais concreta do estilo de vestuário, ainda não garante um "ajuste perfeito" (CHUN, 2007). Porém, o futuro dos sistemas de dimensionamento em massa "feitos sob medida" dependerá da capacidade de produzir, criar e distribuir roupas de qualidade e na aceitação de novos modelos de compra de vestuário pelo consumidor, que por sua vez dependerá dos avanços e desenvolvimento de tecnologias como escaneamento corporal, design assistido por computador e sistemas de fabricação assistidos por computador, experiência virtual, etc. (PETROVA, 2007).

Fig. 4 - Simuladores que utilizam realidade aumentada.
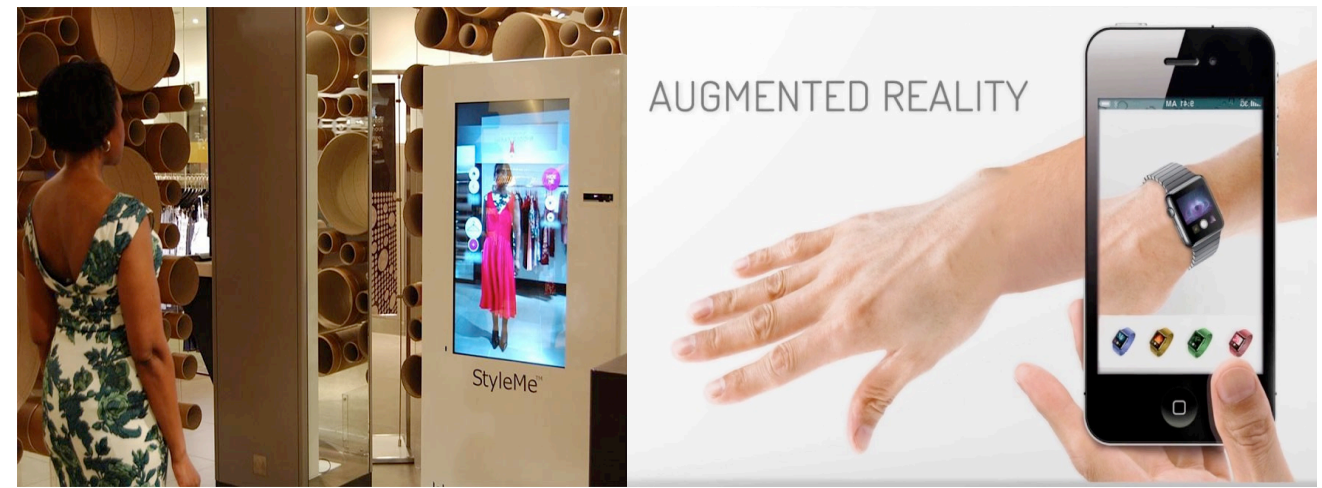

Fonte: https://www.orangemantratech.com/ blog/augmented-reality-takes-fashion-industry-next-level/.

Loker (2007) prevê outra oportunidade futura para o dimensionamento personalizado em massa que pode envolver o ajuste das peças ready-to-wear ${ }^{2}$ existentes. Usando as ferramentas de visualização que estão sendo desenvolvidas, os padrões de ready-to-wear podem ser "embrulhados" no escaneamento corporal de um indivíduo, ajustando-se as modelagens em 2D à peça de roupa item que são em 3D. O consumidor ou empresa poderia avaliar o conteúdo e ajustar os padrões para o tamanho existente para aquele consumidor precisamente. A avaliação visual, em vez da comparação de medições de padrões lineares, seria usada para verificar o material de vestuário e, em seguida, as tecnologias de habilitação ajustariam os padrões existentes. Outra opção seria incluir ajustes de tamanho no design da roupa que poderiam ser modificados após a compra. As variações de comprimento e plenitude são ajustes de tamanho pós-compra óbvios. A autora conclui: o advento das visualizações virtuais de vestuário deu inicio a uma oportunidade para melhorar o ajuste e seu entendimento tanto por parte dos profissionais quanto dos consumidores.

A maior reclamação dos consumidores de peças de roupas on-line está relacionada às

\footnotetext{
${ }^{2}$ Ready-to-wear: (masc. e fem.) Período: meio do século 20 em diante. O termo em inglês para prèt-â-porter (francês); as coleções baratas desenhadas por marcas de moda em todo o mundo. No entanto, o termo é frequentemente aplicado a todas as roupas compradas prontas (CUMMING; CUNNINGTON; CUNNINGTON, 2010). O segundo significado será adotado neste artigo.
} 
medidas e ao ajuste das mesmas. Por causa da falta de padronização das medidas as marcas adotam as tabelas que julgam atender melhor seu público alvo. Isso gera tabelas diferentes para cada marca causando confusão e insegurança em quem compra. As interfaces do futuro podem trazer soluções, para se não eliminar, ao menos mitigar o problema.

O processo de compra nem sempre é racional; pode envolver questões subjetivas culturais e emocionais (JEDDI et al., 2013); proporciona valores relacionados ao cumprimento de tarefas assim como valores hedônicos por meio de respostas que são obtidas durante essa experiência (BABIN; DARDEN; GRIFFIN, 1994). Não é possível projetar-se uma experiência, somente projetarse em função dela, designers podem criar possibilidades, mas não certezas. Afirmar que um grupo de recomendações sempre produzirá determinadas emoções, corre-se o risco de ir-se mais além do que poderá ser cumprido (HASSENZAHL, 2004).

Até o momento, não existe uma medida de UX geral que seja aceita amplamente, mas pode ser avaliada de muitas maneiras diferentes (ROTO et al., 2010). Para escolher-se um método de avaliação da UX é necessário saber de antemão quantos participantes são necessários e quais métricas irá usar-se (TULLIS; ALBERT, 2013) e então elaborar um plano de pesquisa que deve conter os seguintes itens:

1) As perguntas que quer responder; 2) Por que é importante respondê-las; 3) As técnicas que irá usar para respondê-las; 4) Os recursos disponíveis (tempo, orçamento, pessoas, equipamento); e 4) Quando e onde irá acontecer e quem irá realizar (GOODMAN; KUNIAVSKY; MOED, 2012).

Por causa da complexidade da experiência do usuário e todos os aspectos envolvidos, o processo de avaliação não é uma tarefa simples e direta (MASHAPA; VAN GREUNEN, 2010), devese planejar antes de ir à campo interagir com os usuários. Como mencionado acima, existem diversas técnicas de pesquisa que podem ser aplicadas para medir a UX. Farrell (2017); lista algumas categorizadas por objetivo:

Descobrir: Estudo de campo, Diários, Entrevista com o usuário, Entrevista com stakeholders, Coleta de requisitos e restrições;

Explorar: Análise da competição, Revisão de design, Construção de persona, Análise de tarefa, Mapeamento da jornada, Protótipo de feedback e teste, Escrever histórias de usuários, Card sorting;

Testar: Teste de usabilidade qualitativo, Benchmark, Avaliação de acessibilidade; e

Escutar: Survey, Revisão de analytics, Análise de Search-log, Revisão de Usability-bug, Revisão de Frequently-asked-questions (FAQ).

O site All about UX, lista 86 técnicas categorizadas por:

Tipo: estudo de campo, estudo em laboratório, estudos on-line; questionários e escalas Fase de desenvolvimento: cenários, rascunhos (conceitos), primeiros protótipos, protótipos funcionais e produto no mercado

O período da experiência sendo estudada: Antes do uso, durante a interação (partes dela), a experiência de uma tarefa ou atividade e UX de longo prazo

Avaliador/ Provedor da informação: especialistas em UX, um usuário de cada vez, grupo de usuários, pares de usuários (ALL ABOUT UX, [S.d.]).

Objetivando averiguar quais as técnicas de pesquisa são as mais utilizadas para avaliação da UX, Padovani, Schlemmer e Scariot (2012) realizaram uma revisão da literatura e puderam verificar que uma série de métodos utilizados para avaliação de usabilidade também vêm sendo 
empregados para UX (avaliação de co-descoberta; avaliação; etnográfica longitudinal; diário; focus group; repertory grid; shadowing; storytelling workshop). Este dado revela não só a falta de uma definição consolidada do que é UX e como o quanto é confundida com usabilidade. Porém, há de perceber-se que são conceitos distintos (ROTO et al., 2010).

Os autores também encontraram na literatura métodos e técnicas sendo aplicados especificamente para análise da UX, os mais citados na literatura foram:

Attrakdiff (HASSENZAHL; BURMESTER; KOLLER, 2003); Emocards (VERMEEREN et al., 2010);

Reaction Cards (BENEDEK; MINER, 2003); Experience Sampling Method - ESM - solicitação não antecipada de realização de tarefa à distância e feedback imediato do usuário, geralmente aplicado à análise de dispositivos móveis (VERMEEREN et al., 2010).

Futuramente, quando houver uma consolidação do termo, surgirão mais formas específicas para medir a UX. Possivelmente, especulando-se, o uso de mapas da atividade cerebral do usuário para verificar quais partes do cérebro entram em ação ao comprar on-line e desta forma fazer associações às experiências positivas e negativas de compras no sítios testados.

\section{Conclusão}

Neste artigo foi apresentado o cenário geral do e-commerce nacional e mundial com foco no vestuário. Abordou-se o processo decisório de compra do consumidor e como as novas tecnologias estão moldando e modificando a forma como produtos e serviços são comprados e vendidos. Discutiram-se os atributos não-digitais, tais como: as informações de ajuste e caimento de peças de roupas e como a estratégia omnichannel pode dar suporte aos consumidores na busca de informações acerca de produtos e serviços que tenham estes atributos, possibilitando o consumidor a tomar decisões mais assertivas e consequentemente proporcionar uma UX de qualidade. Foram apresentadas as interações atuais do e-commerce com o usuário, quais são as suas interfaces e como as tecnologias em desenvolvimento estão proporcionando recursos para tentar comunicar os atributos não-digitais de vestuário. Por fim, foram listadas e discutidas técnicas de pesquisa que buscam avaliar a UX ao interagir com produtos e interfaces digitais.

\section{Referências}

ABNT. NBR 9241-11: Requisitos ergonômicos para trabalho de escritório com computadores. Parte 11: Orientações sobre usabilidade. . Rio de Janeiro: ABNT, 2002.

ALL ABOUT UX. All evaluation methods. Disponível em: http://www.allaboutux.org/

ANDERSON, Eric T.; HANSEN, Karsten; SIMESTER, Duncan. The Option Value of Returns: Theory and Empirical Evidence. Marketing Science, v. 28, n. 3, p. 405-423, 2009.

ATLAS. e-commerce radar - Moda e Acessórios $1^{\circ}$ semestre 2017. São Paulo: [s.n.], 2017.

BABIN, Barry J.; DARDEN, William R.; GRIFFIN, Mitch. Work and/or fun: Utilitarian shopping value. Journal of Consumer Research, v. 20, n. 4, p. 644-657, 1994.

BECK, Norbert; RYGL, David. Categorization of multiple channel retailing in Multi-, Cross-, and Omni-Channel Retailing for retailers and retailing. Journal of Retailing and Consumer Services, v. 27, p. 170-178, 2015. 
BELL, David. Localização (ainda) é tudo: como vender mais usando a influência do mundo real sobre os hábitos de compra na internet. 1. ed. São Paulo: HSM do Brasil, 2016.

BELL, David R.; GALLINO, Santiago; MORENO, Antonio. Offline Showrooms in Omnichannel Retail: Demand and Operational Benefits. Management Science, p. 1-43, 2016.

CHUN, Jongsuk. Comunication of sizing and fit. In: ASHDOWN, SUSAN P. (Org.). .Sizing in clothing: developing effective sizing systems for ready-to-wear clothing. 1. ed. Cambridge: Woodhead Publishing, 2007. p. 220-243.

COMSCORE. Conferência e-commerce Brasil 2016. . Rio de Janeiro: [s.n.], jan. 2016.

CUMMING, Valerie; CUNNINGTON, C. Willet; CUNNINGTON, Phylis. The dictionary of fashion history. 1. ed. Oxford: Berg Publishers, 2010.

CYBIS, W; HOLTZ, A; FAUST, R. Ergonomia e usabilidade. São Paulo: Novatec, 2010.

E-BIT. $37^{a}$ Edição Relatório Webshoppers. São Paulo: [s.n.]. , mar. 2018.

E-BIT. $36^{a}$ Edição Relatório Webshoppers. São Paulo: [s.n.]. , ago. 2017.

E-COMMERCE NEWS. Ebit: Com forte expansão das compras via mobile, faturamento do ecommerce cresce 10,3\% na Black Friday. Disponível em:

$<$ https://ecommercenews.com.br/noticias/balancos/ebit-com-forte-expansao-das-compras-viamobile-faturamento-do-e-commerce-cresce-103-na-black-friday/>. Acesso em: 7 dez. 2017.

ECOMMERCE FOUNDATION. Global B2C e-commerce report 2016. . Amsterdam: [s.n.], 2016.

FARRELL, Susan. UX research cheat sheet. Disponível em: <https://www.nngroup.com/articles/uxresearch-cheat-sheet/>. Acesso em: 21 out. 2017.

FLAHERTY, Kim. Customer journeys and omnichannel user experience.

FLAHERTY, Kim. Seamless in the omnichannel user experience. Disponivel em:

<https://www.nngroup.com/articles/seamless-cross-channel/>. Acesso em: 26 ago. 2017.

GOODMAN, Elizabeth; KUNIAVSKY, Mike; MOED, Andrea. Observing the user experience. 2. ed. Waltham: Morgan Kaufman, 2012.

GOOGLE. The new multi-screen world: understanding cross-platform consumer behavior. Santa Clara: [s.n.], ago. 2012.

HASSENZAHL, Marc. Emotions can be quite ephemeral; we cannot design them. Interactions, v. 11, n. 5, p. 46, 2004.

HASSENZAHL, Marc; BURMESTER, Michael; KOLLER, Franz. Mensch \& Computer 2003. Interaktion in Bewegung, p. 187-196, 2003.

JEDDI, Shahrzad et al. Consumer behavior and Consumer buying decision process. International Journal of Business and Behavioral Sciences, v. 3, n. 5, p. 20-23, 2013.

JUANEDA-AYENSA, Emma; MOSQUERA, Ana; MURILLO, Yolanda Sierra. Omnichannel customer behavior: Key drivers of technology acceptance and use and their effects on purchase intention. Frontiers in Psychology, v. 7, n. JUL, p. 1-11, 2016.

KATAWETAWARAKS, Chayapa; WANG, Cheng Lu. Online shopper behavior: influences of online shopping decision. v. 1, n. 2, p. 66-74, 2011. 
KOTLER, Philip. Administração de Marketing. 10. ed. São Paulo: Prentice Hall, 2000.

KOTLER, Philip; KELLER, Kevin Lane. Marketing management. 15th Globa ed. Boston: Pearson, 2016.

KPMG. The truth about online consumers: 2017 global online consumer report. New York: [s.n.], jan. 2017.

KUNIAVSKY, Mike. Smart Things, Ubiquitous Computing User Experience Design. Burlington: Morgan Kaufman, 2010.

LAL, Rajiv; SARVARY, Miklos. When and How is the Internet Likely to Decrease Price Competition? Marketing Science, v. 18, n. 4, p. 485-503, 1999.

LOKER, Suzanne. Mass customization and sizing. In: ASHDOWN, SUSAN P (Org.). .Sizing in clothing: developing effective sizing systems for ready-to-wear clothing. 1. ed. Cambridge: Woodhead Publishing, 2007. p. 246-262.

MASHAPA, Job; VAN GREUNEN, Darelle. User experience evaluation metrics for usable accounting tools. Proceedings of the 2010 Annual Research Conference of the South African Institute of Computer Scientists and Information Technologists on - SAICSIT '10, p. 170-181, 2010.

MOCZARNY, I M; DE VILLERS, M R; VAN BILON, J A. How can usability contribute to user experience ? A study in the domain of e-commerce. Saicsit 12, p. 216-225, 2012.

NORMAN, Donald A.; NIELSEN, Jakob. The definition of User Experience. Disponível em: <https://www.nngroup.com/articles/definition-user-experience/>. Acesso em: 15 out. 2016.

PADOVANI, Stephania; SCHLEMMER, Andre; SCARIOT, Cristiele Adriana. Usabilidade \& User Experience, Usabilidade Versus User Experience, Usabilidade Em User Experience? Uma Discussão Teórico-Metodológica Sobre Comunalidades E Diferenças. $12^{\circ}$ ERGODESIGN: $12^{\circ}$ Congresso Internacional de Ergonomia e Usabilidade de Interfaces Humano-Computador., 2012.

PETROVA, Adriana. Creating sizing systems. In: ASHDOWN, SUSAN P. (Org.). Sizing in clothing: developing effective sizing systems for ready-to-wear clothing. 1. ed. Cambridge: Woodhead Publishing, 2007. p. 57-87.

ROTO, Virpi et al. User Experience White Paper: Bringing clarity to the concept of user experience. Seminar on Demarcating User Experience, p. 12, 2010.

SHOPIFY. Fashion \& apparel, cosmetic, jewelry and luxury industry report. Ottawa: [s.n.], 2016.

SOLOMON, Michael R. Comportamento do Consumidor: Comprando, posuindo, sendo. 9. ed. Porto Alegre: Bookman, 2011.

THE NIELSEN COMPANY. Global connected commerce report: what's in store for online grocery shopping. New York: [s.n.], 2017.

TULLIS, Tom; ALBERT, Bill. Measuring the user experience: collecting, analyzing, and presenting usability metrics. 2. ed. Burlington: Morgan Kaufman, 2013.

VERHOEF, Peter C.; KANNAN, P. K.; INMAN, J. Jeffrey. From Multi-Channel Retailing to OmniChannel Retailing. Introduction to the Special Issue on Multi-Channel Retailing. Journal of Retailing, v. 91, n. 2, p. 174-181, 2015. 